\title{
sun. March Scientific Program
}

\section{Plenary Lectures $\quad$ Room 1}

PL4 8:25 - 9:10 Chairperson: Kazuwa Nakao Department of Medicine and Clinical Science, Kyoto University Graduate School of Medicine, Japan

Discovery of nitric oxide and cyclic GMP in cellular signaling

and their role in drug development

Ferid Murad University of Texas Health Science Center, USA

PL5 16:10 - 16:55 Chairperson: Masatomo Mori Department of Medicine and Molecular Science, Gunma University Graduate School of Medicine, Japan

Physiology and pathophysiology of prostanoid receptors

Shuh Narumiya Kyoto University Graduate School of Medicine, Japan

PL6 16:55 - 17:40 Chairperson: Shunichi Yamashita Department of Molecular Medicine, Atomic Bomb Disease Institute, Graduate School of Biomedical Sciences, Nagasaki University, Japan

Molecular and clinical aspects of thyroid hormone transporters

Theo J. Visser Erasmus MC, The Netherlands

\section{Meet-the-Professor $\quad$ 11:10-12:00}

MTP11* Translational research in RAS

Room 2

Chairpersons: Detlev Ganten Charité Foundation, Berlin, Germany

Tadashi Inagami Vanderbilt University School of Medicine, USA

MTP11-1 A mouse model for pregnancy-associated hypertension induced by the "human" renin-angiotensin system

Akiyoshi Fukamizu Center for Tsukuba Advanced Research Alliance (TARA), University of Tsukuba, Japan

MTP11-2 Translational research and the renin angiotensin system

Detlev Ganten Charité Foundation, Berlin, Germany

MTP12 Cushing's syndrome

Chairpersons: André Lacroix Department of Medicine, The University of Montreal Hospital Centre (CHUM), Canada Toshihiro Suda Hirosaki University Graduate School of Medicine, Aomori, Japan

MTP12-1 ACTH-dependent Cushing's syndrome

Yukio Hirata Department of Clinical and Molecular Endocrinology, Tokyo Medical and Dental University, Tokyo, Japan

MTP12-2 Adrenal Cushing's syndrome

André Lacroix Department of Medicine, The University of Montreal Hospital Centre (CHUM), Canada

MTP13 Mineralocorticoid hypertension

Chairpersons: Paul M. Stewart Endocrinology, Clinical and Experimental Medicine, University of Birmingham, Birmingham, UK Ryoyu Takeda Kanazawa University, Ishikawa, Japan

MTP13-1 Mineralocorticoid receptor, salt-sensitive hypertension and metabolic syndrome Toshiro Fujita Department of Nephrology and Endocrinology, University of Tokyo, Tokyo, Japan

MTP13-2 Mineralocorticoid hypertension

Paul M. Stewart Endocrinology, Clinical and Experimental Medicine, University of Birmingham, Birmingham, UK

MTP14* Novel therapies for diabetes: Insulin and beyond

Chairpersons: Leon A. Bach Department of Endocrinology and Diabetes, The Alfred Hospital, Melbourne, Australia Atsunori Kashiwagi Shiga University of Medical Science Hospital, Shiga, Japan

MTP14-1 Incretins and diabetes

Nobuya Inagaki Department of Diabetes and Clinical Nutrition, Graduate School of Medicine, Kyoto University, Kyoto, Japan

MTP14-2 Insulin-like growth factors, diabetes and metabolism

Leon A. Bach Department of Endocrinology and Diabetes, The Alfred Hospital, Melbourne, Australia 


\section{Meet-the-Professor $\quad 11: 10-12: 00$}

\section{MTP15 TSH-secreting pituitary adenoma}

Chairpersons: Paolo Beck-Peccoz University of Milan, Fondazione Ca' Granda IRCCS, Milan, Italy Toshiaki Sano University of Tokushima School of Medicine, Tokushima, Japan

MTP15-1 TSH-secreting pituitary adenoma: Pathology Robert Y. Osamura Tokai University School of Medicine, Kanagawa, Japan

MTP15-2 TSH-secreting pituitary adenomas Paolo Beck-Peccoz University of Milan, Fondazione Ca' Granda IRCCS, Milan, Italy

\section{MTP16 Statins and diabetes}

Chairpersons: D. John Betteridge University College London, UK Hideshi Kuzuya Higashiyama Takeda Hospital, Kyoto, Japan

MTP16-1 Lipid metabolism in type 2 diabetes mellitus and statin therapy Nobuhiro Yamada University of Tsukuba, Tsukuba, Japan

MTP16-2 Diabetes: The need for intensive statin therapy D. John Betteridge University College London, UK

\section{MTP17 Osteoporosis}

Chairpersons: John P. Bilezikian College of Physicians and Surgeons, Columbia University, USA Sung-Kil Lim Division of Endocrinology, Yonsei University College of Medicine, Seoul, Republic of Korea

MTP17-1 Management of osteoporosis: The Japanese guidelines and topics of secondary osteoporosis Ryoichi Takayanagi Department of Medicine and Bioregulatory Science, Kyushu University, Fukuoka, Japan

MTP17-2 Advances in the treatment of osteoporosis John P. Bilezikian College of Physicians and Surgeons, Columbia University, USA

\section{MTP18 Polycystic ovary syndrome}

Chairpersons: Stephen Franks Institute of Reproductive and Developmental Biology, Imperial College London, UK Hirohisa Kurachi Department of Obstetrics and Gynecology, Yamagata University Faculty of Medicine, Yamagata, Japan

MTP18-1 New Criteria of polcystic ovary syndrome in Japan Minoru Irahara The University of Tokushima, Tokushima, Japan

MTP18-2 Polycystic ovary syndrome

Stephen Franks Institute of Reproductive and Developmental Biology, Imperial College London, UK

MTP19*Gonadotropin regulation

Chairpersons: Margaret A. Shupnik Department of Medicine, University of Virginia, Charlottesville, VA, USA Yasuo Sakuma Nippon Medical School, Tokyo, Japan

MTP19-1 Regulation of gonadotropin receptor Takashi Minegishi Department of Obstetrics and Gynecology, Gunma University Graduate School of Medicine, Japan

MTP19-2 Gonadotropin regulation by GnRH pulses and steroids Margaret A. Shupnik Department of Medicine, University of Virginia, Charlottesville, VA, USA

Chairpersons: Jeffrey F. Moley Washington University School of Medicine, USA Hiroshi Takami Department of Surgery, Teikyo University School of Medicine, Tokyo, Japan

MTP20-1 Treatment of medullary thyroid carcinoma based on germline RET mutation analysis Akira Miyauchi Department of Surgery, Kuma Hospital, Kobe, Japan

MTP20-2 Recent advances in diagnosis and treatment Jeffrey F. Moley Washington University School of Medicine, USA

Chairpersons: Elaine M. F. Costa The Developmental Endocrine Unit, Clinical Hospital, University of Sao Paulo, Sao Paulo, Brazil Toshiaki Tanaka Tanaka Growth Clinic, Japan

MTP21-1 Genetics of DSD (disorders of sex development)

Tsutomu Ogata National Research Institute for Child Health and Development, Japan

MTP21-2 Disorders of sex development

Elaine M.F. Costa The Developmental Endocrine Unit, Clinical Hospital, University of Sao Paulo, Sao Paulo, Brazil 


\section{Symposia 9:20-11:00}

Chairpersons: Takashi Kadowaki Department of Metabolic Diseases, Graduate School of Medicine, The University of Tokyo, Tokyo, Japan

Roger J. Davis Howard Hughes Medical Institute and University of Massachusetts Medical School, USA

SY 1-5-1 * Role of adiponectin /AdipoR1 in skeletal muscle in mitochondrial biogenesis, exercise endurance and insulin sensitivity Takashi Kadowaki Department of Metabolic Diseases, Graduate School of Medicine, The University of Tokyo, Tokyo, Japan

SY 1-5-2 Metabolic harmony via neuronal information highways Hideki Katagiri Center for Translational and Advanced Animal Research, Tohoku University Graduate School of Medicine, Sendai, Japan

SY 1-5-3 Role of JNK1 in diet-induced obesity and insulin resistance Roger J. Davis Howard Hughes Medical Institute and University of Massachusetts Medical School, USA

SY 1-5-4 Insulin action and resistance in the central nervous system Jens C. Brüning Institute for Genetics, University of Cologne, Germany

\section{SY1-9 Type 1 diabetes}

Chairpersons: Linda S. Wicker Department of Medical Genetics, Cambridge Institute for Medical Research, University of Cambridge, UK Toshiaki Hanafusa First Department of Internal Medicine, Osaka Medical College, Osaka, Japan

SY 1-9-1 * Type 1 diabetes susceptibility genes: Expression and functional variations determined by genotype Linda S. Wicker Department of Medical Genetics, Cambridge Institute for Medical Research, University of Cambridge, UK

SY 1-9-2 Zinc transporter 8 (SLC30A8), an insulin secretory granule protein with links to the pathogenesis of type 1 and type 2 diabetes John C. Hutton Barbara Davis Center for Childhood Diabetes, Anschutz Medical Center, University of Colorado Denver, Denver, USA

SY 1-9-3 Immune therapy of autoimmune diabetes: The use of CD3 monoclonal antibodies Lucienne Chatenoud INSERM U580, Faculté Paris Descartes, Paris, France

SY 1-9-4 Fulminant type 1 diabetes: An important subtype within type 1 diabetes Toshiaki Hanafusa First Department of Internal Medicine, Osaka Medical College, Osaka, Japan

SY2-6 New treatment of diabetes and obesity

Chairpersons: Rudolph L. Leibel Columbia University, USA Hironobu Yoshimatsu Department of Internal Medicine I, Faculty of Medicine, Oita University, Oita, Japan

SY 2-6-1 * Incretin-based therapies: Mechanisms of action, benefits, and risks Daniel Drucker Mt. Sinai Hospital, Samuel Lunenfeld Research Institute, University of Toronto, Canada

SY 2-6-2 Energy homeostasis in the weight-reduced state Rudolph L. Leibel Columbia University, USA

SY 2-6-3 Hypothalamic neuronal histamine as therapeutic target for the treatment of obesity Hironobu Yoshimatsu Department of Internal Medicine I, Faculty of Medicine, Oita University, Oita, Japan

SY 2-6-4 ER stress, chemical cahperones and insulin sensitivity Umut Ozcan Children's Hospital Boston, Harvard Medical School, USA

\section{SY4-2 Advanced aspects of thyroid carcinoma}

Chairpersons: Matthew D. Ringel Department of Internal Medicine, The Ohio State University, USA Toru Takano Department of Laboratory Medicine, Osaka University Graduate School of Medicine, Japan

SY 4-2-1 * Targeting invasive thyroid cancers: Defining targets and designing therapies Matthew D. Ringel Department of Internal Medicine, The Ohio State University, USA

SY 4-2-2 Diversity of oncogenic BRAFV600E pathways in differentiated papillary thyroid cancer Minho Shong Department of Internal Medicine, Chungnam National University School of Medicine, Daejeon, Republic of Korea

SY 4-2-3 Fetal cell microchimerism in thyroid cancer Laura Fugazzola Endocrine Unit, Fondazione Policlinico IRCCS, Milan, Italy

SY 4-2-4 Advanced aspects of thyroid carcinoma: Genetic bases for targeting the MAP kinase and PI3K pathways Mingzhao Xing Division of Endocrinology and Metabolism, The Johns Hopkins University School of Medicine, USA 


\section{Symposia 9:20-11:00}

\section{SY4-7 Radiation and thyroid diseases}

Chairpersons: Shunichi Yamashita Department of Molecular Medicine, Atomic Bomb Disease Institute, Graduate School of Biomedical Sciences, Nagasaki University, Nagasaki, Japan Arthur B. Schneider College of Medicine, University of Illinois at Chicago, Chicago, Illinois, USA

SY 4-7-1 * Molecular genetics of radiation-induced thyroid cancers Shunichi Yamashita Department of Molecular Medicine, Atomic Bomb Disease Institute, Graduate School of Biomedical Sciences, Nagasaki University, Nagasaki, Japan

SY 4-7-2 The Chernobyl Tissue Bank: A model for integrating research on single blocks of tissue Geraldine A. Thomas Human Cancer Studies Group, Imperial College London, UK

SY 4-7-3 Radiogenic and sporadic papillary thyroid carcinoma after Chernobyl: A comparative morphological analysis Alexander Abrosimov Endocrinological Research Center, Moscow, Russia

SY 4-7-4 Radiation and thyroid disease as seen from the clinic

Arthur B. Schneider College of Medicine, University of Illinois at Chicago, Chicago, Illinois, USA

SY5-3 PTH

Chairpersons: T. John Martin St Vincent's Institute of Medical Research, Australia

Norio Amizuka Department of Developmental Biology of Hard Tissue, Graduate School of Dental Medicine, Hokkaido University, Japan

SY 5-3-1 * PTH mechanisms in anabolic action

T. John Martin St Vincent's Institute of Medical Research, Australia

SY 5-3-2 PTH/PTHrP receptor-mediated actions of parathyroid hormone (PTH) in kidney and bone

Harald Jueppner Endocrine Unit and Pediatric Nephrology Unit, Massachusetts General Hospital and Harvard Medical School, USA

SY 5-3-3 Histological assessment for PTH/PTHrP signaling on osteoblasts

Norio Amizuka Department of Developmental Biology of Hard Tissue, Graduate School of Dental Medicine, Hokkaido University, Japan

SY 5-3-4 PTH and the skeleton: Lessons from knock-out mice

Andrew C. Karaplis Lady Davis Institute for Medical Research, McGill University, Montreal, Canada

\section{SY7-2 Hypothalamic AMPK for controlling energy metabolism}

Chairpersons: David Carling MRC Clinical Sciences Centre, Imperial College London, UK Yasuhiko Minokoshi Division of Endocrinology and Metabolism, National Institute for Physiological Sciences, Okazaki, Japan

SY 7-2-1* The role of the AMPK cascade in regulating energy metabolism David Carling MRC Clinical Sciences Centre, Imperial College London, UK

SY 7-2-2 Role of the paraventricular hypothalamic AMP kinase in food selection behavior Yasuhiko Minokoshi Division of Endocrinology and Metabolism, National Institute for Physiological Sciences, Okazaki, Japan

SY 7-2-3 Malonyl-COA and CPT1c regulation of body weight Michael J. Wolfgang Department of Biological Chemistry, Johns Hopkins University, USA

SY 7-2-4 Hypothalamic angiopoietin like peptide-4 is a novel regulator of body weight homeostasis Min-Seon Kim Department of Internal Medicine, University of Ulsan College of Medicine, Seoul, Republic of Korea

\section{SY8-1 Aromatase}

Chairpersons: Angela Brodie Department of Pharmacology and Experimental Therapeutics, School of Medicine and the Greenebaum Cancer Center, University of Maryland, Baltimore, MD, USA Makio Shozu Department of Reproductive Medicine, Graduate School of Medicine, Chiba University, Chiba, Japan

SY 8-1-1 * Mechanism-based strategies for extending benefits of aromatase inhibitor treatment for breast cancer Angela Brodie Department of Pharmacology and Experimental Therapeutics, School of Medicine and the Greenebaum Cancer Center, University of Maryland, Baltimore, MD, USA

SY 8-1-2 Organization-function relationship of CYP19A1 promoters Makio Shozu Department of Reproductive Medicine, Graduate School of Medicine, Chiba University, Chiba, Japan

SY 8-1-3 Mechanisms in the regulation of aromatase/CYP19A1 gene expression during human trophoblast differentiation Carole R. Mendelson Departments of Biochemistry and Obstetrics \& Gynecology, University of Texas Southwestern Medical Center, Dallas, Texas, USA

SY 8-1-4 X-ray structure of human aromatase: What makes this P450 unique? Debashis Ghosh Hauptman-Woodward Institute and Roswell Park Cancer Institute, USA 


\section{Symposia 9:20-11:00}

* State-of- the-art

SY8-11 Rapid (nongenomic) action of steroid receptor

Room 12

Chairpersons: Cheryl S. Watson Department of Biochemistry and Molecular Biology, University of Texas Medical Branch, Galveston, Texas, USA

Ellis R. Levin University of California Irvine and the Long Beach VA Medical Center, USA

SY 8-11-1 * Xenoestrogen and estrogen actions via membrane receptor-initiated signaling pathways in pituitary, neuronal, and breast cancer cells Cheryl S. Watson Department of Biochemistry and Molecular Biology, University of Texas Medical Branch, Galveston, Texas, USA

SY 8-11-2 Extra-nuclear estrogen receptors

Ellis R. Levin University of California Irvine and the Long Beach VA Medical Center, USA

SY 8-11-3 Novel aspects of signaling activation by steroid hormones in target cells

Gabriella Castoria Department of General Pathology, Second University of Naples, Italy

SY 8-11-4 MAP kinase inputs to progesterone receptor action in breast cancer models

Carol A. Lange Departments of Medicine and Pharmacology, University of Minnesota, Minneapolis, MN, USA

SY10-3 Neuroendocrine tumors

Chairpersons: Kjell E. Öberg Department of Endocrine Oncology, Uppsala University Hospital, Uppsala, Sweden

Guido Rindi Università Cattolica del Sacro Cuore, Policlinico A.Gemelli, Rome, Italy

SY 10-3-1* Neuroendocrine tumors - Current diagnostic and therapeutic strategies

Kjell E. Öberg Department of Endocrine Oncology, Uppsala University Hospital, Uppsala, Sweden

SY 10-3-2 Pathology classification of gastroenteropancreatic (GEP) neuroendocrine tumors (NET)

Guido Rindi Università Cattolica del Sacro Cuore, Policlinico A.Gemelli, Rome, Italy

SY 10-3-3 TBD

Akira Shimatsu National Hospital Organization Kyoto Medical Center, Kyoto, Japan

SY 10-3-4 Current status and therapeutic strategy for gastroenteropancreatic neuroendocrine tumors in Japan

Tetsuhide Ito Department of Medicine and Bioregulatory Science, Graduate School of Medical Sciences, Kyushu University, Fukuoka, Japan

SY11-6 Novel signaling mechanisms of ovarian function

Room 11

Chairpersons: JoAnne S. Richards Department of Molecular and Cellular Biology, Baylor College of Medicine, Houston, TX, USA

Kaoru Miyamoto Department of Biochemistry, Faculty of Medical Sciences, University of Fukui, Fukui, Japan

SY 11-6-1* Mediators of LH action during ovulation

JoAnne S. Richards Department of Molecular and Cellular Biology, Baylor College of Medicine, Houston, TX, USA

SY 11-6-2 Molecular mechanisms underlying the activation of mammalian primordial follicles

Kui Liu Department of Medical Biochemistry and Biophysics, Umea University, Sweden

SY 11-6-3 Mechanism of stem cell differentiation into steroidogenic lineage

Kaoru Miyamoto Department of Biochemistry, Faculty of Medical Sciences, University of Fukui, Fukui, Japan

SY 11-6-4 Inter-follicle signalling and the regulation of initiation of follicle growth

Kate Hardy Jaroslav Stark and Stephen Franks, Imperial College London, UK

SY12-3 In vitro conversion of stem cells to beta cells

Chairpersons: Alberto Hayek Department of Pediatrics, Pediatric Diabetes Research Center, University of California, San Diego, USA

Shoen Kume Institute of Molecular Embryology and Genetics, Kumamoto University, Kumamoto, Japan

SY 12-3-1* In vitro conversion of stem cells to beta cells

Alberto Hayek Department of Pediatrics, Pediatric Diabetes Research Center, University of California, San Diego, USA

SY 12-3-2 The guided differentiation of ES cells into the pancreatic lineage

Shoen Kume Institute of Molecular Embryology and Genetics, Kumamoto University, Kumamoto, Japan

SY 12-3-3 TBD

Hongkui Deng College of Life Sciences, Peking University, China

SY 12-3-4 Beta-cell regeneration: Pancreatic endocrine stem cells and high-throughput screening

Fred Levine Burnham Institute for Medical Research, San Diego, USA 


\section{Symposia 9:20-11:00}

Chairpersons: Benjamin G. Neel Ontario Cancer Institute, Canada

Eisuke Nishida Department of Cell and Developmental Biology, Graduate School of Biostudies, Kyoto University, Kyoto, Japan

SY 14-3-1 * Mechanism of action of Noonan syndrome-associated Raf mutation Benjamin G. Neel Ontario Cancer Institute, Canada

SY 14-3-2 Roles of the MAP kinase signaling pathways in the regulation of life span Eisuke Nishida Department of Cell and Developmental Biology, Graduate School of Biostudies, Kyoto University, Kyoto, Japan

SY 14-3-3 S6K1: A determinant in embryonic-stem-cell commitment during early adipocyte differentiation George Thomas Department of Cancer and Cell Biology, Metabolic Diseases Institute, College of Medicine, University of Cincinnati, USA

SY 14-3-4 Regulation and function of the TSC-mTOR pathway Kun-Liang Guan Department of Pharmacology and Moores Cancer Center, University of California San Diego, La Jolla, CA, USA

SY15-1 Mineralocorticoid receptor and hypertension

Chairpersons: Celso E. Gomez-Sanchez G.V. Montgomery VA Medical Center and University of Mississippi, USA Hirotaka Shibata Department of Internal Medicine, Keio University School of Medicine, Japan

SY 15-1-1 * Mineralocorticoid receptor and hypertension Celso Gomez-Sanchez G.V. Montgomery VA Medical Center and University of Mississippi, USA

SY 15-1-2 Cell-specific regulation of mineralocorticoid receptor signalling in cardiovascular disease Morag J. Young Prince Henry's Institute of Medical Research, Australia

SY 15-1-3 Mineralocorticoid receptor (MR)-related hypertension: Alternative pathways of MR transactivation Hirotaka Shibata Department of Internal Medicine, Keio University School of Medicine, Japan

SY 15-1-4 Mechanisms of aldosterone autonomy - Insights from animal models

Felix Beuschlein Medizinische Klinik Innenstadt, Ludwig-Maximilians University in Munich, Germany

\section{SY15-4 New aspect of CAH/adrenal insufficiency}

Chairpersons: Richard J.M. Ross Department of Human Metabolism, University of Sheffield, Medical School, UK Deborah P. Merke National Institutes of Health, Bethesda, Maryland, USA

SY 15-4-1* Current health status of adult patients with congenital adrenal hyperplasia and the potential for new therapies Richard Ross Department of Human Metabolism, University of Sheffield, Medical School, UK

SY 15-4-2 Therapeutic management of adrenal insufficiency Wiebke Arlt Centre for Endocrinology, Diabetes and Metabolism, School of Clinical and Experimental Medicine, University of Birmingham, Birmingham,UK

SY 15-4-3 New aspects of congenital adrenal hyperplasia Deborah P. Merke National Institutes of Health, Bethesda, Maryland, USA

SY 15-4-4 The metabolic impact of improving diurnal cortisol exposure in patients with adrenal insufficiency Gudmundur Johannsson Department of Endocrinology, Sahlgrenska Academy, University of Gothenburg, Sweden 


\section{Symposia 14:20-16:00}

Chairpersons: Stephen 0'Rahilly University of Cambridge Metabolic Research Laboratories, Institute of Metabolic Science, Addenbrookes Hospital, Cambridge, UK Hiroaki Masuzaki Second Department of Internal Medicine, Faculty of Medicine, University of the Ryukyus, Okinawa, Japan

SY 2-1-1 * Molecular mechanisms in human obesity \& insulin resistance Stephen O'Rahilly University of Cambridge Metabolic Research Laboratories, Institute of Metabolic Science, Addenbrookes Hospital, Cambridge, UK

SY 2-1-2 The role of frequent and rare DNA variation in obesity Philippe Froguel Department of Genomics of Common Diseases, Imperial College London and CNRS 8199, Pasteur Institute, Lille, France

SY 2-1-3 The molecular pathophysiology of a human obesity syndrome

Val C. Sheffield Department of Pediatrics, Howard Hughes Medical Institute, University of lowa, lowa City, IA, USA

SY 2-1-4 FTO and obesity

Ulrich Ruther Institute for Animal Developmental and Molecular Biology, Heinrich Heine University, Dusseldorf, Germany

\section{SY3-1 Ghrelin: Recent progress}

Chairpersons: Kenji Kangawa National Cardiovascular Center Research Institute, Osaka, Japan Tamas L. Horvath Section of Comparative Medicine, Yale University School of Medicine, New Haven, USA

SY 3-1-1* Ghrelin: From discovery to translational research Kenji Kangawa National Cardiovascular Center Research Institute, Osaka, Japan

SY 3-1-2 Phenotypic analysis of ghrelin knockout mouse Masayasu Kojima Institute of Life Science, Kurume University, Fukuoka, Japan

SY 3-1-3 Ghrelin regulates neuronal function and synaptic plasticity in a mitochondrial redox-dependent manner Tamas L. Horvath Section of Comparative Medicine, Yale University School of Medicine, New Haven, USA

SY 3-1-4 Ghrelin and its receptor (GHS-R): Recent progress

Roy G. Smith The Department of Metabolism and Aging, The Scripps Research Institute, Jupiter, Florida, USA

\section{SY3-6 New technology in endocrine research}

Chairpersons: Ronald G. Blasberg Departments of Neurology and Radiology, Memorial Hospital, Molecular Pharmacology \& Chemistry Program, Sloan-Kettering Institute, Memorial Sloan-Kettering Cancer Center (MSKCC), Zuckerman Research Center (ZRC), USA Mitsutoshi Setou Department of Molecular Anatomy, Hamamatsu University School of Medicine, Shizuoka, Japan

SY 3-6-1* Molecular-genetic imaging: The hedgehog signaling pathway in endocrine gland development and disease Ronald G. Blasberg Departments of Neurology and Radiology, Memorial Hospital, Molecular Pharmacology \& Chemistry Program, Sloan-Kettering Institute, Memorial Sloan-Kettering Cancer Center (MSKCC), Zuckerman Research Center (ZRC), USA

SY 3-6-2 Progress and challenges in neuroendocrine and neural crest tumours: Molecular imaging and therapy Giovanni Lucignani Department of biomedical sciences and technologies, University of Milan, Italy

SY 3-6-3 TBD

Mark W. Duncan University of Colorado Denver, USA

SY 3-6-4 Development and endocrinological applications of mass microscope

Mitsutoshi Setou Department of Molecular Anatomy, Hamamatsu University School of Medicine, Shizuoka, Japan

SY4-1 Advances in thyroid hormone action

Chairpersons: Mitsushige Nishikawa The Second Department of Internal Medicine, Kansai Medical University, Osaka, Japan Eun Jig Lee Department of Internal Medicine, Division of Endocrionology, Yonsei University College of Medicine, Seoul, Korea

SY 4-1-1 * Thyroid hormone action: Overview Mitsushige Nishikawa The Second Department of Internal Medicine, Kansai Medical University, Osaka, Japan

SY 4-1-2 Role of thyroid hormone receptor TRalpha in atherosclerosis development Jacques Samarut Institute of Functional Genomics, Ecole Normale Supérieure de Lyon, France

SY 4-1-3 Reduced sensitivity to TH: An expanded view of RTH Samuel Refetoff Departments of Medicine, Pediatrics and Genetics, The University of Chicago, Chicago, Illinois, USA

SY 4-1-4 Aberrant histone modifications involved in resistance to thyroid hormone Masanobu Yamada Department of Medicine and Molecular Science, Gunma University Graduate School of Medicine, Maebashi, Japan 


\section{Symposia 14:20-16:00}

SY5-2 VDR

Room 9

Chairpersons: Daniel D. Bikle Departments of Medicine and Dermatology, VA Medical Center, University of California, San Francisco, CA, USA

Yasuhiro Takeuchi Department of Endocrinology, Endocrine Center, Toranomon Hospital, Tokyo, Japan

SY 5-2-1 * Differential regulation of epidermal function by VDR coactivators

Daniel D. Bikle Departments of Medicine and Dermatology, VA Medical Center, University of California, San Francisco, CA, USA

SY 5-2-2 A new bone-active vitamin D compound for fracture prevention in osteoporosis

Toshio Matsumoto Department of Medicine and Bioregulatory Sciences, University of Tokushima Graduate School of Medical Sciences, Tokushima, Japan

SY 5-2-3 TBD

Ritsuko Masuyama School of Dentistry, Nagasaki University, Nagasaki, Japan

SY 5-2-4 Actions of the vitamin D receptor in the skin

Marie B. Demay Endocrine Unit, Massachusetts General Hospital, Harvard Medical School, Boston, MA, USA

\section{SY7-1 Clinical update on acromegaly}

Room 4

Chairpersons: Kazuo Chihara Hyogo Prefectural Kakogawa Medical Center, Japan

Susan M. Webb IIB-Sant Pau and Department of Endocrinology/Medicine, Hospital Sant Pau, UAB and Centro de Investigación Biomédica en Red de Enfermedades Raras (CIBER-ER, Unidad 747), Instituto de Salud Calros III, Spain

SY 7-1-1* Pathophysiology of acromegaly: Progression of aging process by IGF-I excess?

Kazuo Chihara Hyogo Prefectural Kakogawa Medical Center, Japan; Department of Internal Medicine, Division on Diabetes, Metabolism and Endocrinology, Kobe University Graduate School of Medicine, Japan

SY 7-1-2 Long-term outcome in treated acromegaly

Susan M. Webb IIB-Sant Pau and Department of Endocrinology/Medicine, Hospital Sant Pau, UAB and Centro de Investigación Biomédica en Red de Enfermedades Raras (CIBER-ER, Unidad 747), Instituto de Salud Calros III, Spain

SY 7-1-3 The combination of medical treatments in acromegaly

Aart J. van der Lely Department of Medicine, Erasmus University MC, Rotterdam, The Netherlands

SY 7-1-4 Complicated osteoporosis is highly prevalent in patients with acromegaly

Andrea Giustina Department of Medical and Surgical Sciences, University of Brescia, Brescia, Italy

\section{SY8-6 NR and biology}

Room 5

Chairpersons: Daniel Metzger IGBMC, INSERM U964, CNRS UMR7104, University of Strasbourg, Illkirch, France

Juro Sakai Division of Metabolic Medicine, Research Center for Advanced Science and Technology, University of Tokyo, Japan

SY 8-6-1 * Control of mouse energy metabolism by the nuclear receptor PPARß and the transcriptional co-regulator TIF2 in skeletal muscle myocytes Daniel Metzger IGBMC, INSERM U964, CNRS UMR7104, University of Strasbourg, Illkirch, France

SY 8-6-2 Genetic models of androgen regulation of testis and ovary

David J. Handelsman ANZAC Research Institute, University of Sydney, Sydney, Australia

SY 8-6-3 Metabolic neuronal pathways autonomic nervous system involved in inter-organ communication Yoshitomo Oka Division of Molecular Metabolism and Diabetes, Department of Internal Medicine, Tohoku University, Japan

SY 8-6-4 Obesity and metabolic syndrome in histone demethylase JHDM2a deficient mice Juro Sakai Division of Metabolic Medicine, Research Center for Advanced Science and Technology, University of Tokyo, Japan

SY9-2 New paradigm of the renin-angiotensin system

Chairpersons: Carlos M. Ferrario Hypertension and Vascular Disease Center, Wake Forest University School of Medicine, USA

Masatsugu Horiuchi Department of Molecular Cardiovascular Biology and Pharmacology, Ehime University Graduate School of Medicine, Japan

SY 9-2-1* Harmonic complexity within the renin angiotensin system: Role of the Ang-(1-7)/ACE2/mas receptor axis Carlos M. Ferrario Hypertension and Vascular Disease Center, Wake Forest University School of Medicine, USA

SY 9-2-2 Roles of angiotensin II type 2 receptor and its associated protein, ATIP in anti-aging Masatsugu Horiuchi Department of Molecular Cardiovascular Biology and Pharmacology, Ehime University Graduate School of Medicine, Japan

SY 9-2-3 N-acetyl-seryl-aspartyl-lysyl-proline (AC-SDKP), a tetrapeptide: Role in target organ damage (TOD) in hypertension Oscar A. Carretero Department of Internal Medicine, Hypertension and Vascular Research, Henry Ford Hospital, Detroit, MI, USA

SY 9-2-4 New paradigm of the renin-angiotensin system: Cardiometabolic implications Robson Santos Federal University of Minas Gerais, Brazil 


\section{Symposia 14:20-16:00}

Chairpersons: Ryozo Nagai Department of Cardiovascular Medicine, University of Tokyo Graduate School of Medicine, Japan Guo-Ping Shi Department of Medicine, Brigham and Women's Hospital and Harvard Medical School, USA

SY 9-3-1 * Interplay among the vascular, metabolic and immune systems in cardiovascular remodeling and metabolic syndrome Ryozo Nagai Department of Cardiovascular Medicine, University of Tokyo Graduate School of Medicine, Japan

SY 9-3-2 Cardiovascular hormones play dual roles in oxygen/energy handling in 'Metabolic Domino' Hiroshi Itoh Department of Internal Medicine, Keio University School of Medicine, Japan

SY 9-3-3 Mast cell function in cardiovascular diseases and metabolic disorders Guo-Ping Shi Department of Medicine, Brigham and Women's Hospital and Harvard Medical School, USA

SY 9-3-4 Control of endothelial progenitor cell function, endothelial function and vascularization capacity in mice and humans by endocrine signaling Thomas Thum Institute for Molecular and Translational Therapeutic Strategies, Hannover Medical School, Germany

Chairpersons: Jeff Holly Department of Clinical Science at North Bristol, University of Bristol, UK Atsushi Ochiai Pathology Division, Research Center for Innovative Oncology, National Cancer Center Hospital East, Japan

SY 10-5-1* IGFs and cancer Jeff Holly Department of Clinical Science at North Bristol, University of Bristol, UK

SY 10-5-2 Role of bone-derived IGFs on human prostate cancer bone metastasis Atsushi Ochiai Pathology Division, Research Center for Innovative Oncology, National Cancer Center Hospital East, Japan

SY 10-5-3 Insulin-like growth factor binding protein-2 (IGFBP-2) action in cancer cells Vincenzo C. Russo Centre for Hormone Research, Murdoch Children's Research Institute, Royal Children's Hospital; and Department of Paediatrics, University of Melbourne, Australia

SY 10-5-4 Growth factors and estrogen signaling implicated in the hormonal therapy for human breast cancer Shin-ichi Hayashi Department of Molecular and Functional Dynamics, Tohoku University Graduate School of Medicine, Japan; Research Institute for Clinical Oncology, Saitama Cancer Center, Japan

\section{SY11-7 Endocrine aspects of andrology}

Chairpersons: Frederick Wu Andrology Research Unit, Manchester Academic Health Science Centre, Central Manchester University Hospitals Foundation Trust, UK Gianni Forti Endocrine Unit, Department of Physiopathology, University of Florence, Italy

SY 11-7-1 * Endocrinology of the ageing male - The European Male Ageing Study (EMAS) Frederick C.W. Wu Andrology Research Unit, Manchester Academic Health Science Centre, Central Manchester University Hospitals Foundation Trust, UK

SY 11-7-2 Detailed studies of seminal germ cell morphology and semen biochemistry are very valuable tools for diagnosis and management of azoospermic subjects

Somnath Roy National Institute of Health and Family Welfare, New Delhi, India

SY 11-7-3 Androgen deficiency in older men: What do we really know? Ronald Swerdloff Los Angeles Biomedical Research Institute at Harbor-UCLA Medical Center, USA

SY 11-7-4 Mechanisms of androgen action on the skeletal muscle Shalender Bhasin Boston University School of Medicine, USA

SY12-4 In vivo conversion of progenitor cells to beta cells

Chairpersons: Bernard Portha B2PE Lab, Paris Diderot University, Paris, France Hideto Kojima Department of Molecular Genetics in Medicine, Shiga University of Medical Science, Shiga, Japan

SY 12-4-1 * In vivo conversion of progenitor cells to beta cells: An adaptive response to increased insulin demand which serves as a basis for novel therapeutic strategies Bernard Portha B2PE Lab, Paris Diderot University, Paris, France

SY 12-4-2 Molecular control of human beta cell replication Andrew F. Stewart Division of Endocrinology, University of Pittsburgh, Pittsburgh, Pennsylvania, USA

SY 12-4-3 Liver-targeting pancreatic islet regeneration by helper-dependent adenovirus Hideto Kojima Department of Molecular Genetics in Medicine, Shiga University of Medical Science, Shiga, Japan

SY 12-4-4 In vivo conversion of progenitor cells to beta cells Ahmed Mansouri Department of Molecular Cell Biology, Molecular Cell Differentiation Group, Max-Planck Institute for Biophysical Chemistry, Germany 
Chairpersons: Ole H. Petersen School of Biosciences, Cardiff University, Cardiff, UK Naoaki Saito Biosignal Research Center, Kobe University, Japan

SY 14-1-1* Intracellular imaging of $\mathrm{Ca}^{2+}$, calmodulin, trypsin and STIM1 in a polarized cell Ole H. Petersen School of Biosciences, Cardiff University, Cardiff, UK

SY 14-1-2 PKC targeting and its involvement in the pathogenesis of spinocerebellar ataxia Naoaki Saito Biosignal Research Center, Kobe University, Japan

SY 14-1-3 Imaging signaling from G-protein-coupled receptors to CAMP Martin J. Lohse Institute of Pharmacology and Toxicology, University of Würzburg; Rudolf Virchow Center, University of Wurzburg, Wurzburg, Germany

SY 14-1-4 Secretagogue-induced multiple signal interconnections in insulin secreting cells Hideo Mogami Department of Physiology, Hamamatsu University School of Medicine, Japan

SY15-5 Pheochromocytoma

Chairpersons: Graeme Eisenhofer Institute of Clinical Chemistry and Laboratory Medicine and Department of Medicine, University Hospital Carl Gustav Carus, Dresden, Germany Anne-Paule Gimenez-Roqueplo Paris Descartes University, Paris, France; INSERM, UMR970, Paris, France

SY 15-5-1 * Pheochromocytomas and paragangliomas

Graeme Eisenhofer Institute of Clinical Chemistry and Laboratory Medicine and Department of Medicine, University Hospital Carl Gustav Carus, Dresden, Germany

SY 15-5-2 Genetics and genomics of paraganglioma and pheochromocytoma

Anne-Paule Gimenez-Roqueplo Paris Descartes University, Paris, France; INSERM, UMR970, Paris, France

SY 15-5-3 Pheochromocytoma: An update in Japan

Kazuhiro Takekoshi University of Tsukuba, Japan

SY 15-5-4 TBD

Akihiro Sakurai Shinshu University School of Medicine, Nagano, Japan 


\section{JES-Sponsored Symposia}

JES4 Up-to-date in endocrine hypertension

9:20-11:00 Room 16

Chairpersons: William F. Young Jr. Mayo Clinic College of Medicine, Rochester, USA Yukio Hirata Department of Clinical and Molecular Endocrinology, Tokyo Medical and Dental University, Tokyo, Japan

JES4-1 Up-to-date in endocrine hypertension Gian Paolo D. Rossi DMCS Internal Medicine 4, University of Padua, Italy

JES4-2 Revisiting the confirmatory tests in the diagnosis of primary aldosteronism Mitsuhide Naruse Department of Endocrinology, Metabolism and Hypertension, National Hospital Organization Kyoto Medical Center, Japan

JES4-3 Pheochromocytoma in Peking Union Medical College Hospital of China

Zhengpei Zeng Department of Endocrinology,Peking Union Medical College Hospital, Beijing, China

JES4-4 Endocrine hypertension

Xavier Bertagna Hôpital Cochin, Faculté de Médecine Paris Descartes, Paris, France

JES5 Novel functions of angiotensin receptors

9:20-11:00 Room 17

Chairpersons: Carlos M. Ferrario Wake Forest University School of Medicine, USA Akiyoshi Fukamizu University of Tsukuba, Japan

JES5-1 Actions of glomerular AT1 angiotensin receptors to promote kidney injury and inflammation Thomas M. Coffman Department of Medicine, Division of Nephrology, Duke University Medical Center, Durham, NC, USA

JES5-2 Host stromal angiotensin type 1a receptor signaling regulates tumor-associated angiogenesis during tumor growth Masataka Majima Department of Pharmacology, Kitasato University School of Medicine, Kanagawa, Japan

JES5-3 Role of renin-angiotensin system in metabolic syndrome Masaru Iwai Department of Molecular Cardiovascular Biology and Pharmacology, Ehime University Graduate School of Medicine, Ehime, Japan

JES5-4 Angiotensin receptors signaling in cardiac remodeling Koichiro Kuwahara Department of Medicine and Clinical Science, Kyoto University Graduate School of Medicine, Kyoto, Japan

JES6 Hypoadiponectinemia -upstream and downstream

Chairpersons: Gerald Reaven Stanford University School of Medicine, USA lichiro Shimomura Osaka University Graduate School of Medicine, Osaka, Japan

JES6-1 Ectopic fat deposition and lipotoxic disorders Michio Shimabukuro Second Department of Internal Medicine, Faculty of Medicine, University of the Ryukyus, Okinawa, Japan

JES6-2 TBD Umut Ozcan Harvard Medical School, USA

JES6-3 Physiological and pathophysiological roles of adiponectin and AdipoRs Toshimasa Yamauchi Department of Metabolic Diseases, University of Tokyo, Tokyo, Japan

JES6-4 Physiological function of adiponectin and the remedy for hypoadiponectinemia Norikazu Maeda Department of Metabolic Medicine, Graduate School of Medicine, Osaka University, Osaka, Japan

JES7 Molecular aspects of endocrine tumors update

Chairpersons: Kennichi Kakudo Department of Pathology, Wakayama Medical University, Wakayama, Japan Mitsuhide Naruse Department of Endocrinology, Metabolism and Hypertension, National Hospital Organization Kyoto Medical Center, Kyoto, Japan

JES7-1 Stem cells in thyroid carcinomas Shioko Kimura Laboratory of Metabolism, National Cancer Institute, National Institutes of Health, Bethesda, MD, USA

JES7-2 Thyroid transcription factor-1 (TTF-1) in thyroid cancers and its application to therapeutics Ryohei Katoh Department of Human Pathology, University of Yamanashi, Japan

JES7-3 Heritable gastroenteropancreatic NET: Diagnostic aspects and EU classification guidelines Guido Rindi Università Cattolica del Sacro Cuore, Policlinico A.Gemelli, Rome, Italy

JES7-4 Pituitary lesions in genetic endocrine tumor syndromes Sylvia L. Asa University Health Network, Ontario Cancer Institute, University of Toronto, Ontario, Canada 


\section{Oral Hot Topics 12:10-13:00}

\begin{tabular}{|c|c|c|}
\hline OH5 & Diabetes mellitus 2 & Room 9 \\
\hline \\
\hline & OH5-1 OH5-2 OH5 & \\
\hline
\end{tabular}

OH6 Obesity and adiposcience 1

Room 10

Chairperson: lichiro Shimomura Department of Metabolic Medicine (Internal Medicine), Graduate School of Medicine, Osaka University, Japan

OH6-1 OH6-2 OH6-3

OH7 Pituitary / Neuroendocrino
$\begin{gathered}\text { Chairperson: Miyuki Ishibashi Tsukune Clinic, Japan } \\ \text { OH7-1 OH7-2 OH7-3 }\end{gathered}$

OH8 Steroid receptors Room 12

Chairperson: Noriyuki Koibuchi Department of Integrative Physiology, Gunma University Graduate School of Medicine, Japan

$\mathrm{OH} 8-1 \quad \mathrm{OH} 8-2 \quad \mathrm{OH} 8-3$

\section{Oral Sessions 11:10-12:00}

$\begin{array}{llll}09 & \text { Diabetes mellitus } 3 & \text { Room } 11 \\ \text { Chairperson: Yuichiro Yamada Akita University Graduate School of Medicine, Japan } \\ \begin{array}{ccccc}\text { O 9-1 } & \text { O 9-2 } & \text { O 9-3 } & \text { O 9-4 }\end{array}\end{array}$

O 10 Obesity and adiposcience 1 Room 12

Chairperson: Kikuo Kasai Dokkyo University School of Medicine, Japan

O 10-1 $\quad$ O 10-2 O 10-3 O 10-4

011 Thyroid 2

Room 13
O 12 Pituitary / Neuroendocrinology 2 Room 16 Chairperson: Tamotsu Shibasaki Department of Physiology, Nippon Medical School, Japan O12-1 $\quad 012-2 \quad 012-3 \quad 012-4$

013 Nuclear receptors Room 17 Chairperson: Hirotaka Shibata Keio University School of Medicine, Japan O 13-1 $013-2 \quad 013-3 \quad 013-4$

\section{Poster Presentations 13:10-14:20 Event Hall}

\section{P1-8 Diabetes mellitus 8}

Chairperson: Masato Kasahara Department of Medicine and Clinical Science, Kyoto University Graduate School of Medicine, Kyoto, Japan

$\begin{array}{lllll}\text { P1-8-1 P1-8-2 } & \text { P1-8-3 P1-8-4 P1-8-5 P1-8-6 }\end{array}$ $\mathrm{P} 1-8-7 \quad \mathrm{P} 1-8-8$

P1-9 Diabetes mellitus 9

Chairperson: Kentaro Doi Rakuwakai Otowa Hospital, Kyoto, Japan

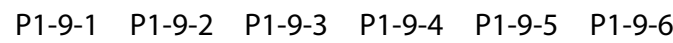

P1-9-7 P1-9-8 P1-9-9

P1-10 Diabetes mellitus 10

Chairperson: Kazuhiko Takaya Department of Diabetes, Endocrinology, and Metabolism, Shizuoka General Hospital, Shizuoka, Japan

P1-10-1 P1-10-2 P1-10-3 P1-10-4 P1-10-5 P1-10-6 P1-10-7 P1-10-8

P1-11 Diabetes mellitus 11

Chairperson: Shinji Susa The Third Department of Internal Medicine, Yamagata University School of Medicine, Japan

P1-11-1 P1-11-2 P1-11-3 P1-11-4 P1-11-5 P1-11-6 $\mathrm{P} 1-11-7$ P1-11-8

P1-12 Diabetes mellitus 12

Chairperson: Hiroyuki Konya Department of Internal Medicine, Ashiya Municipal Hospital, Japan

P1-12-1 P1-12-2 P1-12-3 P1-12-4 P1-12-5 P1-12-6

P1-12-7 P1-12-8
P1-13 Diabetes mellitus 13

Chairperson: Kenji Ueshima EBM Research Center, Kyoto University Graduate School of Medicine, Japan

P1-13-1 P1-13-2 P1-13-3 P1-13-4 P1-13-5 P1-13-6 P1-13-7 P1-13-8

P1-14 Diabetes mellitus 14

Chairperson: Atsushi Suzuki Division of Endocrinology and Metabolism,

Fujita Health University, Aichi, Japan

P1-14-1 P1-14-2 P1-14-3 P1-14-4 P1-14-5 P1-14-6 P1-14-7 P1-14-8

P2-6 Obesity and adiposcience 6

Chairperson: Hiroshi Arai Department of Medicine and Clinical Science, Kyoto University Graduate School of Medicine, Kyoto, Japan

P2-6-1 P2-6-2 P2-6-3 P2-6-4 P2-6-5 P2-6-6

P2-6-7 P2-6-8

P2-7 Obesity and adiposcience 7

Chairperson: Michio Noguchi Department of Medicine and Clinical Science, Kyoto University Graduate School of Medicine, Kyoto, Japan

P2-7-1 P2-7-2 P2-7-3 P2-7-4 P2-7-5 P2-7-6

P2-7-7 P2-7-8

P2-8 Obesity and adiposcience 8

Chairperson: Tsutomu Tomita Department of Medicine and Clinical Science, Kyoto University Graduate School of Medicine, Japan

$\begin{array}{llllll}\text { P2-8-1 P2-8-2 } & \text { P2-8-3 P2-8-4 P2-8-5 P2-8-6 }\end{array}$

$P 2-8-7 \quad P 2-8-8$ 
Poster Presentations 13:10-14:20 Event Hall

P2-9 Obesity and adiposcience 9

Chairperson: Hironori Waki Department of Metabolic Diseases, Graduate School of Medicine, The University of Tokyo, Tokyo, Japan

$\begin{array}{llllll}\text { P2-9-1 P2-9-2 P2-9-3 P2-9-4 P2-9-5 P2-9-6 } & \text { P2 }\end{array}$

P2-9-7 P2-9-8

P2-10 Obesity and adiposcience 10

Chairperson: Hiroshi Hirose Health Center, Keio University, Tokyo, Japan P2-10-1 P2-10-2 P2-10-3 P2-10-4 P2-10-5 P2-10-6 P2-10-7 P2-10-8

P3-1 Peptides 1

Chairperson: Goro Katsuura Shionogi, Co., Ltd. and Department of Medicine and Clinical Science, Kyoto University Graduate School of Medicine, Kyoto, Japan P3-1-1 P3-1-2 P3-1-3 P3-1-4 P3-1-5 P3-1-6 P3-1-7 P3-1-8

P4-6 Thyroid 6

Chairperson: Hideo Sugawa Sugawa Clinic, Japan

P4-6-1 P4-6-2 P4-6-4 P4-6-5 P4-6-6 P4-6-7 P4-6-8 P4-6-9

P4-7 Thyroid 7

Chairperson: Kumiko Tsuboi Division of Diabetes, Metabolism and Endocrinology, Department of Medicine, Toho University School of Medicine, Japan

P4-7-1 P4-7-2 P4-7-3 P4-7-4 P4-7-5 P4-7-6 P4-7-7 P4-7-8

P4-8 Thyroid 8

Chairperson: Nobuhiro Fukunari Department of Surgery, Showa University School of Medicine, Northern Yokohama Hospital, Kanagawa, Japan

P4-8-1 P4-8-2 P4-8-3 P4-8-4 P4-8-5 P4-8-6

P4-8-7 P4-8-8 P4-8-9

P4-9 Thyroid 9

Chairperson: Hiroki Shimura The Third Department of Internal Medicine, University of Yamanashi, Yamanashi, Japan

P4-9-1 P4-9-2 P4-9-3 P4-9-4 P4-9-5 P4-9-6

P4-9-7 P4-9-8 P4-9-9

P5-4 Bone and mineral metabolism 4

Chairperson: Ryoichi Takayanagi Department of Medicine and Bioregulatory

Science, Kyushu University, Fukuoka, Japan

P5-4-1 P5-4-2 P5-4-3 P5-4-4 P5-4-5 P5-4-6

P5-4-7 P5-4-8

P5-5 Bone and mineral metabolism 5

Chairperson: Takahiro Okamoto Department of Endocrine Surgery, Tokyo Women's Medical University, Tokyo, Japan

P5-5-1 P5-5-2 P5-5-3 P5-5-4 P5-5-5 P5-5-6

$P 5-5-7 \quad P 5-5-8$

P5-6 Bone and mineral metabolism 6

Chairperson: Hisaya Kawate Department of Medicine and Bioregulatory Science, Graduate School of Medical Sciences, Kyushu University, Fukuoka, Japan

P5-6-1 P5-6-2 P5-6-3 P5-6-4 P5-6-5 P5-6-6 $P 5-6-7$ P5-6-8

P7-6 Pituitary / Neuroendocrinology 6

Chairperson: Yutaka Oki Hamamatsu University School of Medicine, Japan

P7-6-1 P7-6-2 P7-6-3 P7-6-4 P7-6-5 P7-6-6

P7-6-7 P7-6-8
P7-7 Pituitary / Neuroendocrinology 7

Chairperson: Yoshihisa Uenoyama Graduate School of Bioagricultural Sciences, Nagoya University, Nagoya, Japan

P7-7-1 P7-7-2 P7-7-3 P7-7-4 P7-7-5 P7-7-6

P7-7-7 P7-7-8

P7-8 Pituitary / Neuroendocrinology 8

Chairperson: Hiroyuki Ariyasu Translational Research Center, Kyoto University Hospital, Kyoto, Japan

P7-8-1 P7-8-2 P7-8-3 P7-8-4 P7-8-5 P7-8-6

P7-8-7 P7-8-8

P7-9 Pituitary / Neuroendocrinology 9

Chairperson: Yutaka Takahashi Department of Internal Medicine, Division of Diabetes, Metabolism and Endocrinology, Kobe University Graduate School of Medicine,

Japan

$\begin{array}{llllll}\text { P7-9-1 P7-9-2 P7-9-3 P7-9-4 P7-9-5 P7-9-6 } & \text { P7 }\end{array}$

P7-9-7 P7-9-8

P7-10 Pituitary / Neuroendocrinology 10

Chairperson: Shozo Yamada Department of Hypothalamic and Pituitary Surgery, Toranomon Hospital, Japan

P7-10-1 P7-10-2 P7-10-3 P7-10-4 P7-10-5 P7-10-6

P7-10-7 P7-10-8

P8-1 Steroid receptors 1

Chairperson: Takuya Murata Department of Physiology, University of Fukui, Fukui, Japan P8-1-1 P8-1-2 P8-1-3 P8-1-4 P8-1-5 P8-1-6 P8-1-7 P8-1-8 P8-1-9

P9-5 CVEM 5

Chairperson: Kouichi Tamura Department of Medical Science and Cardiorenal Medicine, Yokohama City University Graduate School of Medicine, Japan

P9-5-1 P9-5-2 P9-5-3 P9-5-4 P9-5-5 P9-5-6

$P 9-5-7 \quad P 9-5-8$

P9-6 CVEM 6

Chairperson: Tatsuo Shimosawa Department of Clinical Laboratory, University of Tokyo, Tokyo, Japan

P9-6-1 P9-6-2 P9-6-3 P9-6-4 P9-6-5 P9-6-6

P9-6-7 P9-6-8

P9-7 CVEM 7

Chairperson: Noboru Fukuda Department of Medicine, Division of Nephrology, Hypertension and Endocrinology, Nihon University School of Medicine, Tokyo, Japan

P9-7-1 P9-7-2 P9-7-3 P9-7-4 P9-7-5 P9-7-6 P9-7-7 P9-7-8

P10-3 Tumor 3

Chairperson: Akihiro Sakurai Shinshu University School of Medicine, Japan P10-3-1 P10-3-2 P10-3-3 P10-3-4 P10-3-5 P10-3-6 $P 10-3-7 \quad P 10-3-8$

P10-4 Tumor 4

Chairperson: Atsushi Nakagawa Department of Endocrinology and Metabolism, Kanazawa Medical University, Japan

P10-4-1 P10-4-2 P10-4-3 P10-4-4 P10-4-5 P10-4-6 P10-4-7 P10-4-8 


\section{Poster Presentations 13:10-14:20 Event Hall}

P11-4 Reproduction and development 4

Chairperson: Kenji Ohyama Department of Pediatrics, University of Yamanashi, Japan

P11-4-1 P11-4-2 P11-4-3 P11-4-4 P11-4-5 P11-4-6

P11-4-7 P11-4-8

P11-5 Reproduction and development 5

Chairperson: Toshiya Matsuzaki Department of Obstetrics and Gynecology, Institute of Health Biosciences, The University of Tokushima Graduate School, Japan

P11-5-1 P11-5-2 P11-5-3 P11-5-4 P11-5-5 P11-5-6

P11-5-7 P11-5-8

P11-6 Reproduction and development 6

Chairperson: Maki Fukami Department of Endocrinology and Metabolism, National Research Institute for Child Health and Development, Tokyo, Japan

P11-6-1 P11-6-2 P11-6-3 P11-6-4 P11-6-5 P11-6-6

P11-6-7 P11-6-8

P12-1 Regeneration

Chairperson: Masakatsu Sone Department of Medicine and Clinical Science, Kyoto University Graduate School of Medicine, Japan

P12-1-1 P12-1-2 P12-1-3 P12-1-4 P12-1-5 P12-1-6

P12-1-7 P12-1-8 P12-1-9
P15-4 Adrenal 4

Chairperson: Takeshi Usui Clinical Research Institute for Endocrine and Metabolic Diseases, National Hospital Organization Kyoto Medical Center, Kyoto, Japan

P15-4-1 P15-4-2 P15-4-3 P15-4-4 P15-4-5 P15-4-6

$P 15-4-7 \quad P 15-4-8$

\section{P15-5 Adrenal 5}

Chairperson: Akiyo Tanabe Department of Medicine, Institute of Clinical Endocrinology, Tokyo Women's Medical University, Tokyo, Japan

P15-5-1 P15-5-2 P15-5-3 P15-5-4 P15-5-5 P15-5-6 P15-5-7 P15-5-8

P15-6 Adrenal 6

Chairperson: Isamu Miyamori The Third Department of Internal Medicine, University of Fukui, Fukui, Japan

P15-6-1 P15-6-2 P15-6-3 P15-6-4 P15-6-5 P15-6-6 $P 15-6-7 \quad P 15-6-8 \quad P 15-6-9$

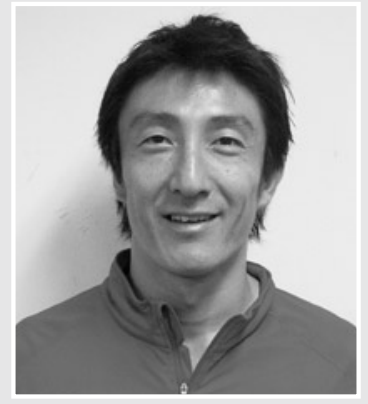

口特別対談

「ホルモンとスポーツ」 Hormone and sports

・ゲスト

北京五輪陸上男子400Mリレー 銅メダリスト

朝原宣治氏

大阪ガス株式会社

Beijing Olympic Games $400 \mathrm{~m}$ relay

bronze medalist

Nobuharu Asahara

Osaka Gas Co., Ltd.

○聞き手

中尾一和 京都大学教授

Kazuwa Nakao Kyoto University

\section{市民公開講座 Public Forum}

\section{3月28日 (日) 14:00-16:30 \\ 国立京都国際会館 Annex1 (収容600名)}

\section{ホルモンと成長・健康・長寿}

Hormone as a key to better growth, health and longevity

口講演 1

加齢と肥満、糖尿病

Obesity and diabetes in the aspect of aging

中尾一和 京都大学教授

Kazuwa Nakao Kyoto University

口講演2

男性更年期

Andropause

松田公志 関西医科大学教授

Tadashi Matsuda Kansai Medical University

\section{$*$}

その場で体操「すわろビクス」

Fitness exercise with a chair

梅田陽子

京都大学医学部附属病院健康運動指導士

Yo-ko Umeda Kyoto University Hospital
口講演3

ホルモンとこどもの 健やかな成長・発達

Healthy growth and development of children and youth

長谷川奉延 慶應義塾大学准教授 Tomonobu Hasegawa Keio University

口講演4

骨粗鬆症

Osteoporosis

松本俊夫 徳島大学教授

Toshio Matsumoto The University of Tokushima

口講演5

脳のホルモンとその病気

Neuroendocrine hormone

and related disease

寺本明 日本医科大学教授

Akira Teramoto Nippon Medical School 
Nippon Boehringer Ingelheim Co., Ltd. / Astellas Pharma Inc.

Chairperson: Masakazu Haneda Asahikawa Medical College, Japan

Speaker: Theodore W. Kurtz University of California, San Francisco, USA

LS7 Long Term Safety of Bisphosphonates

ONO PHARMACEUTICAL CO., LTD

Chairperson: Toshio Matsumoto Department of Medicine and Bioregulatory Sciences, University of Tokushima Graduate School of Medical Science, Japan Speaker: Steven R. Cummings San Francisco Coordinating Center / Medicine and Epidemiology \& Biostatistics (Emeritus), University of California, USA

LS8 Incretin Pathophysiology and Therapy in Type 2 Diabetes

BANYU PHARMACEUTICAL CO., LTD.

Chairperson: Kiminori Hosoda Department of Endocrinology and Metabolism, Graduate School of Medicine, Kyoto University, Japan Speaker: Jack L. Leahy Professor of Medicine, Chief of Endocrinology, Diabetes and Metabolism, University of Vermont, USA

LS9 Mechanisms by which 5-Hydroxytryptamine 2A receptor signaling cascade regulates adiponectin expression

\section{Mitsubishi Tanabe Pharma Corporation}

Chairperson: Yoshihiro Ogawa Department of Molecular Medicine and Metabolism, Medical Research Institute, Tokyo Medical and Dental University, Japan

Speaker: Toshimasa Yamauchi Department of Integrated Molecular Science on Metabolic Diseases,

Department of Metabolic Diseases, Graduate School of Medicine, The University of Tokyo, Japan

LS10 Strategic Options to Reduce its Complications in Treatment of Type 2 Diabetes Mellitus

Novo Nordisk Pharma Ltd.

Chairperson: Nobuya Inagaki Department of Diabetes and Clinical Nutrition, Graduate School of Medicine, Kyoto University, Japan

Speaker: Takashi Kadowaki Department of Diabetes and Metabolic Diseases, Graduate School of Medicine, The University of Tokyo, Japan

LS11 Metabolic Regulation by Endocrine FGFs

Kyowa Hakko Kirin Co., Ltd.

Chairperson: Masashi Mukoyama Department of Medicine and Clinical Science, Kyoto University Graduate School of Medicine, Japan

Speaker: Seiji Fukumoto Department of Medicine, Division of Nephrology and Endocrinology, University of Tokyo Hospital, Japan

\section{Satellite Symposia $\quad$ 17:50-19:20}

SS2 Latest Progress in the Renin-Angiotensin Research

Takeda Pharmaceutical Company Limited

Chairperson: Toshiro Fujita The University of Tokyo, Japan

Speaker: Kenji Ueshima Kyoto University, Japan

Atsuhiro Ichihara Keio University, Japan

Masatsugu Horiuchi Ehime University, Japan

Thomas M. Coffman Department of Medicine, Division of Nephrology, Duke University and Durham Veterans Affairs Medical Centers, USA

Issei Komuro Osaka University / Chiba University, Japan

SS3 Somatostatin analogues: Their current and future role in treating pituitary tumors

\section{Novartis Pharma K.K.}

Chairperson: Akira Shimatsu Clinical Research Institute, National Hospital Organization Kyoto Medical Center, Japan

Steven W.J. Lamberts Erasmus Medical Centre, The Netherlands

Speaker: Akira Shimatsu National Hospital Organization Kyoto Medical Center, Japan

Marily Theodoropoulou Max Planck Institute of Psychiatry, Germany

Steven Lamberts Erasmus Medical Centre, The Netherlands

Rosario Pivonello Federico II University, Italy

Martin Reincke University of Munich, Germany 